\title{
Stitching and Memory-Optimized Processing of Three-Dimensional Riblet Surface Datasets for Oil Channel Tests
}

\author{
Renke Scheuer, Eduard Reithmeier, and Niklas B. Windeler
}

\begin{abstract}
Even modern passenger planes require several tons of kerosene per hour. Therefore, it is a matter of top priority to realize savings for both environmental and economic reasons. One of the approaches towards the reduction of friction losses on airplanes is the structuring of airplane surfaces with riblets. In order to determine the quality of riblet structures, it is necessary to carry out tests of oil passage on upscaled models of the real structures. This paper presents methods which process measurement data from real airplane surfaces and produce memory-optimized CAD data. For this purpose, measurement data of a confocal microscope are used.
\end{abstract}

Index Terms-Confocal microscopy, data reduction, elimination of measurement artifacts, mesh generation, riblets.

\section{INTRODUCTION}

Rising oil prices and a growing focus on the economy are constantly increasing the call for friction-reducing surface structuring. Therefore, already since the 1970s, particularly in the aircraft industry, there has been research work in the area of the so-called riblet structures which, when positioned in the flow direction, reduce the wall friction of turbulent flows. Fig. 1 shows three riblet structures and their drag reduction capacity depending on the Reynolds number.

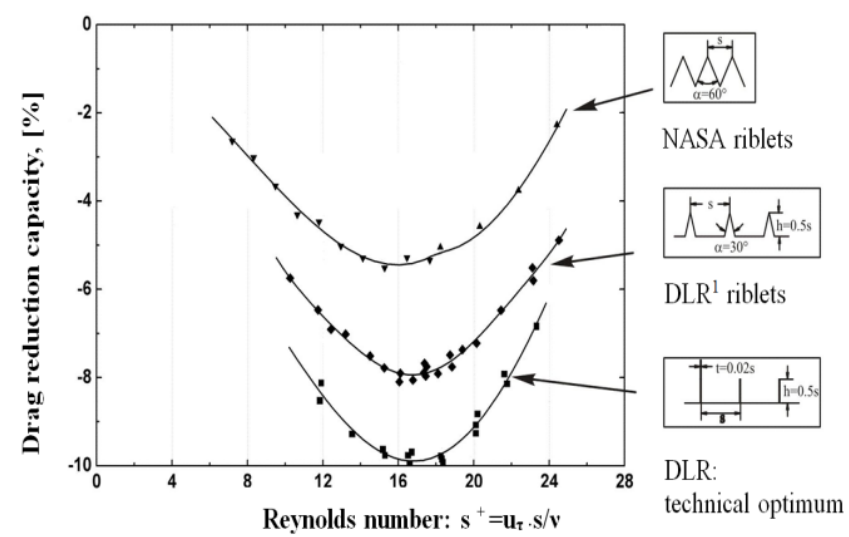

Fig. 1. Drag reduction capacity of various riblet structuresaccording to [1].

Due to their high drag reduction potential and stability, the

Manuscript received June 2, 2013; revised July 31, 2013.

R. Scheuer is with the Institute of Measurement and Automatic Control, Leibniz Universitaet Hannover, Nienburger Straße 1730167 Hanover, Germany (e-mail: renke.scheuer@imr.uni-hannover.de).

E. Reithmeier is the Director of the Institute of Measurement and Automatic Control, Leibniz Universitaet Hannover, Nienburger Straße 17 30167 Hanover, Germany (e-mail: sekretariat@imr.uni-hannover.de).

N. B. Windeler is a Postgraduate Student at the Leibniz Universitaet Hannover (e-mail: sekretariat@imr.uni-hannover.de).
DLR riblets have established themselves as economically advantageous. In the best case, they reduce the friction of turbulent flows up to $8 \%$, compared to a smooth surface. However, the drag reduction significantly depends on the state of the riblets. The drag grows significantly due to an increase of the tip radius [2], abrasion or inaccurate production. The objective of this work is, therefore, to analyze the riblet structures on an airplane wing at different grades of wear and points in time and, on the basis of the obtained data, produce CAD models which can be studied in laboratory conditions. Because measurements on the original object are very difficult to carry out, the impressions of the functional surfaces are measured at any one time, as shown in Fig. 2.

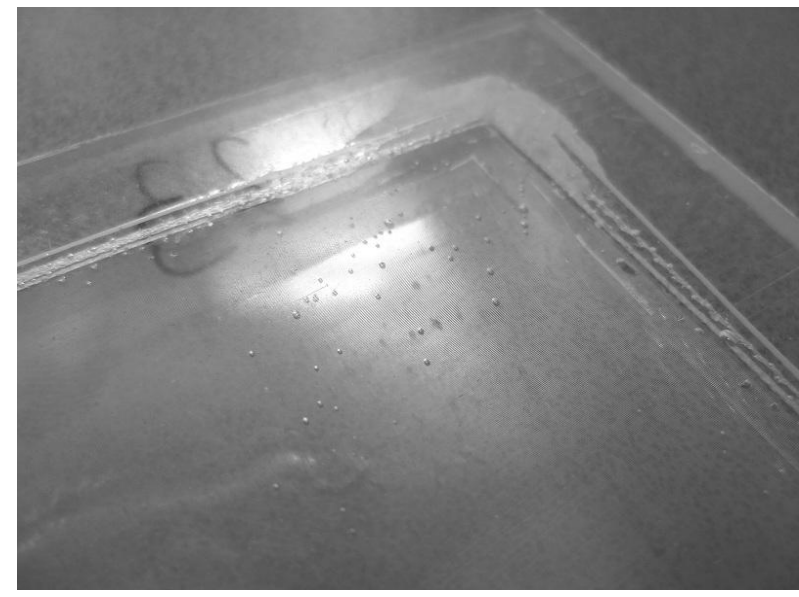

Fig. 2. Impression of the riblet structure to be analyzed.

Especially since the accuracy of the measurements in the fluid air is too low, the measured structures are then upscaled in order for the produced model to be tested in an oil passage. When creating the CAD model, it is also utterly important to pay attention to the data size not to exceed a defined limit.

\section{STITCHING}

The starting position of the measurement and data processing operation is the impression of the functional surface, presented above in Fig. 2. On average, the riblet structures have a period of $100 \mu \mathrm{m}$, are $50 \mu \mathrm{m}$ high and have a tip radius of $1 \mu \mathrm{m}$. In order to collect sets of the measurement data, we have used the areal confocal 3D measurement system ,, Surf " by Nanofocus. A $50 \times$ lens turns out to be the most reasonable to use [3], because it provides the necessary accuracy and also allows measurements of a comparatively large area $(320 \times 320 \mu \mathrm{m})$. 


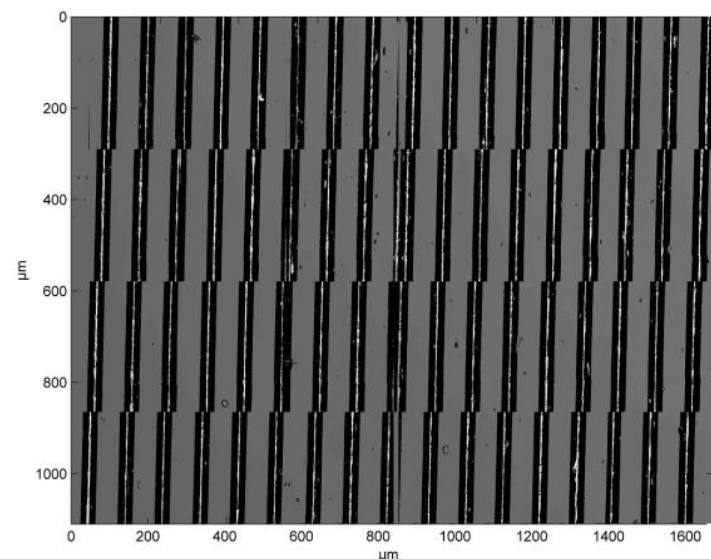

Fig. 3. Measurement data set with stitching errors.

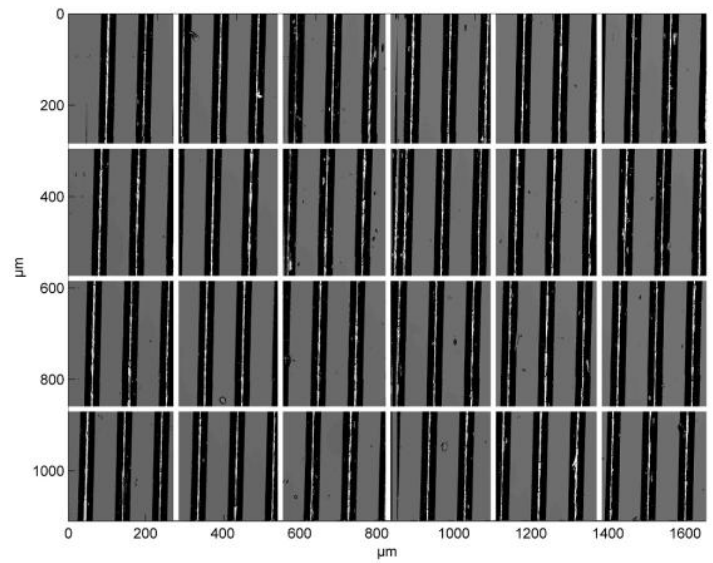

Fig. 4. Measurement data set with marked stitching areas.

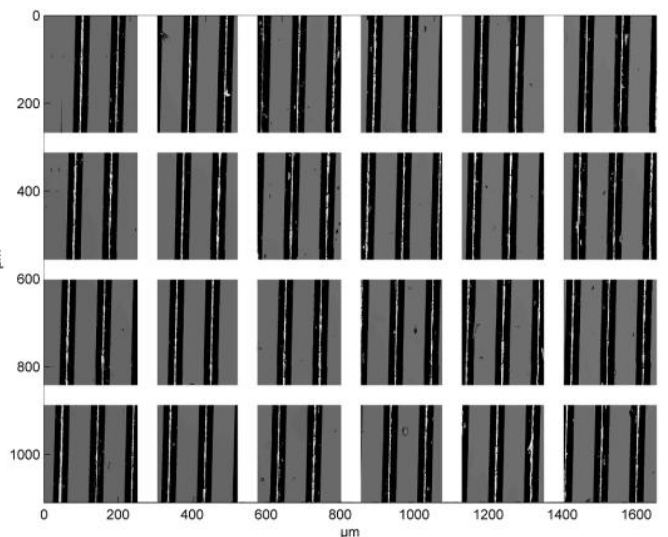

Fig. 5. Measurement data set with areas to be deleted.

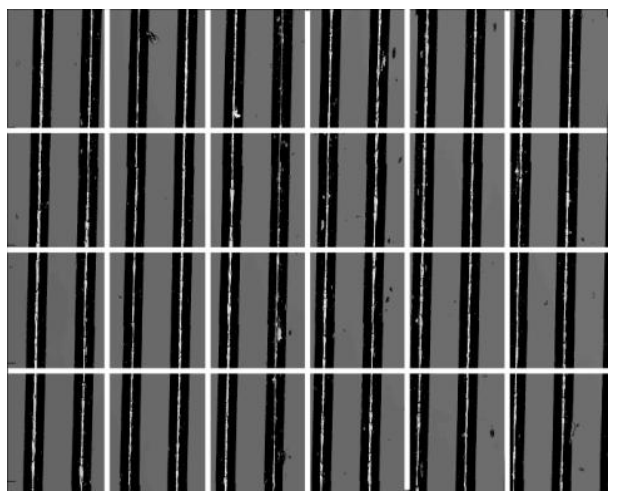

Fig. 6. A new measurement data set with the overlapping area without rough stitching errors.

In order to further increase the measurement range, the software provided by the microscope manufacturer carries out a user defined number of individual measurements with the area of $1024 \times 1024$ pixels. The individual measurements are then stitched by the software program. However, this stitching process is not optimized for recurrent structures; therefore, there is an increasing number of the so-called stitching errors. Fig. 3 shows a measuring field - comprising $6 \times 4$ single images - with stitching errors.

The stitching errors are removed by means of a script. For this reason, all the areas without stitching errors are first determined and extracted. Then these measurement areas are joined with the help of an overlapping area. This creates a measurement data set with fewer measurement data which, however, is joined correctly for the most part. For a better illustration, this process is shown in Fig. 4, Fig. 5 and Fig. 6. Afterwards, the measurement data sets are low-pass filtered and the surface defects are approximated by means of interpolation due to the far too low reflection [4]. In the process, due to the increased accuracy, the Delaunay triangulation [5] algorithm has established itself in contrast to a bilinear surface interpolation. In the end, the riblet structures are arranged vertically and on one level.

So far the data obtained from this data processing operation have been used in order to produce CAD data for oil passage measurements. However, they still have sporadic stitching errors and surface defects due to impurity or artifacts in the positive mold (bubbles). These surface defects, however, can falsify the calculations of the period length during the further procedure and, thus, must be eliminated. Chapter 3 takes a closer look at the detection and substitution process.

\section{Detection OF Surface Defects AND SubStitution}

As already mentioned in Chapter 2, the processed measuring field still has some surface defects which can falsify the result during the further procedure. On the height image, these surface defects characteristically have unrealistically high, low and incorrect values as well as large differences in height in comparison to the adjacent areas. For a better illustration, Fig. 7 and Fig. 8 each show an example of a remaining stitching error and a bubble on the positive mold.

In order to detect defects of this type, two different detection methods are used in the course of the measured value processing. For the first method, at the start the second derivatives in the $\mathrm{x}$ and $\mathrm{y}$ direction are created. Now it is possible to decide for each measured value in the measuring field whether the change of the gradient to the next measured value exceeds the critical threshold. If this is the case, there is a surface defect. This method mainly detects the remaining stitching errors.

Impurities or defects in the positive mold such as bubbles, on the contrary, have a smooth edge profile. Therefore, they are not detected, as it can be seen in Fig. 8. In order to be able to detect these defects as well, a different method is used. First, all measured values are rounded to an integer and then a histogram is created for every column of the measurement data set. By means of the histogram it is now possible to determine the average value and the standard deviation of 
every row. With the help of the calculated standard deviation it is now possible to define an area around the average value. Measured values outside of this area are detected as a part of a surface defect.

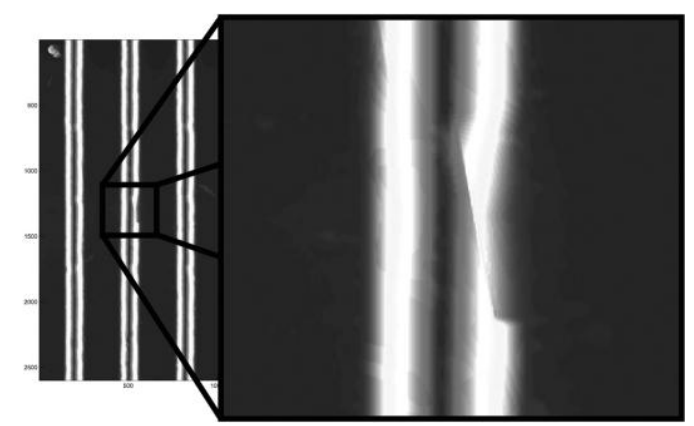

Fig. 7. An example of a stitching error.

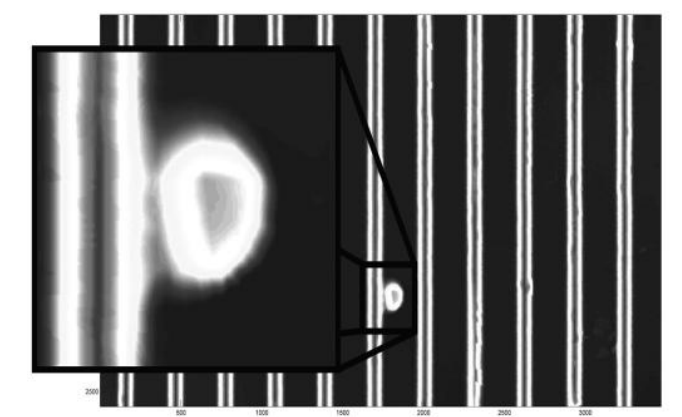

Fig. 8. An example of a positive mold artifact.

After the implementation of the detection method the interrelated surface defects are detected. With the help of their contours it is now possible to define an area which will afterwards be substituted by error-free measured values.

\section{EXTENSION OF THE MEASUREMENT RANGE}

In order to be able to take measurements in the oil passage, it is necessary to have $481 \mathrm{~mm} \times 381 \mathrm{~mm}$ large reproductions of the riblet structure. Due to the scaling, it corresponds to a number of approx. 63 periods. In order to create CAD files, first it is necessary to determine the lengths of period of the measurement data set by means of the Fast Fourier Transformation. Zero padding is used in order to improve the accuracy. Afterwards the measurement data sets are first connected vertically and then horizontally by means of an overlapping area. 6 measurement data sets, respectively, have been used for this application. In doing so, two horizontal rows with three measurement data sets each have been arranged one below the other.

In order for the created matrix to meet the above requirements, the rows of the created matrix are now added vertically to it. The complete matrix is then repeatedly added in the horizontal direction and finally shortened to the desired length. This approach is accepted because the structures have already received a statistically representative measurement by means of the six measurements.

\section{DATA REDUCTION}

After the extension of the measuring range, the created measurement data set contains approx. 70 million height values equidistantly spaced. When using the complete measurement data set, the creation of the CAD model is not possible due to the size. Therefore, it is necessary to reduce the data volume.

Up to now, a square mesh has been used for this purpose. In this method, square areas of a similar height have been saved as a homogeneous surface in the CAD files. This way, it has been possible to represent extensive regions by means of individual squares in flat areas, thus saving disk space. However, surface defects and the bottom area of the riblets are shown in too much detail, which unnecessarily increases the memory requirements. However, we will not go into further detail about this outdated method.

As an alternative to the above method, a new algorithm, based on the geometric primitive triangle, has been developed. In general, triangle meshes are divided into structured and unstructured triangular meshes. Structured meshes have a regular topology and, therefore, a disadvantage that the size of the triangles presents a compromise between the precise image of the detailed areas and the reduction of the number of the triangles. Unstructured triangular meshes do not have a regular topology and are mainly the result of an adaption process. For the further procedure, by means of an unstructured triangular mesh, important areas can be accurately shown in every detail and the total number of triangles can be kept relatively small at the same time. Applied to the riblet structure, it means a very detailed representation of the tips, because, as already mentioned in Chapter 1 , the radius can have a significant influence on the drag reduction potential [2]. However, the mesh size in the area of the sides and the floor can be increased.

In order to carry out preparations for the mesh generation algorithm, the data set with equidistant measured values must be thinned out.

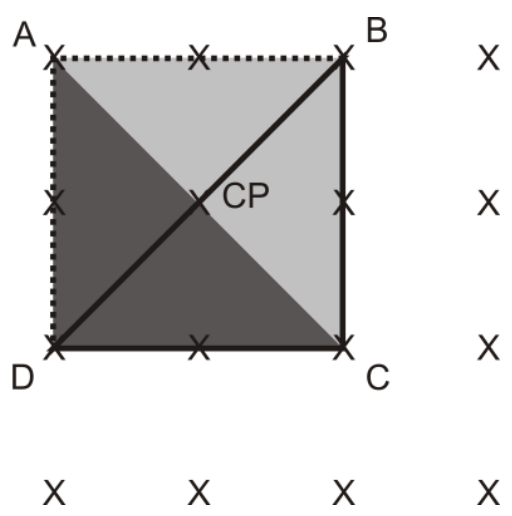

Fig. 9. Measuring points mesh with possible triangles.

In order to reduce the total number of measuring data points, the complete measuring field is analyzed by means of areas sized $3 \times 3$ points. By means of position vectors of corner points, initially four triangles are created: ABC (light grey), ACD (dark grey), BCD (with a black frame) and ABD (with a dotted frame), see Fig. 9.

Afterwards it is necessary to test whether the remaining measuring data (measuring points), situated between the corner points of the triangles or in the centre of the $3 \times 3$ point 
by means of only two triangles respectively, in which they are situated. In order to make sure this is properly done, these points are tested separately as follows. If the distance of the tested measured value to the corresponding triangle exceeds the previously defined threshold, this point cannot be eliminated, because otherwise the created triangle would not be able to describe the geometry with sufficient accuracy. However, it is important to note that if this condition is violated for one point, none of the considered points can be eliminated. Otherwise, during the later triangulation procedure, it could lead to the creation of triangles which would not be considered for the checking of points. However, due to the fact that the partial elimination of points leads to precise results (see Chapter Validation of Results), it is a preferred method for the reasons of saving disk space. The threshold is calculated from the square of the height of the tested measuring point and the gradient in the horizontal direction. The higher the measured value and the current change of the gradient, the more precisely the triangles have to represent the measured value which needs to be substituted. During the examination of the total measuring range by means of areas sized $3 \times 3$ points, we use a single-row overlapping in both horizontal and vertical plane.

According to the same principle, larger areas with $5 \times 5$ points, $9 \times 9$ point and the maximum number of $17 \times 17$ measured values are tested afterwards. The size of the examination area is shown in Fig. 10. In doing so, every subsequent step contains four fields of the previous step. After the complete method has been applied, the obtained measurement data set has only important, unstructured measured values for the representation of the surface.

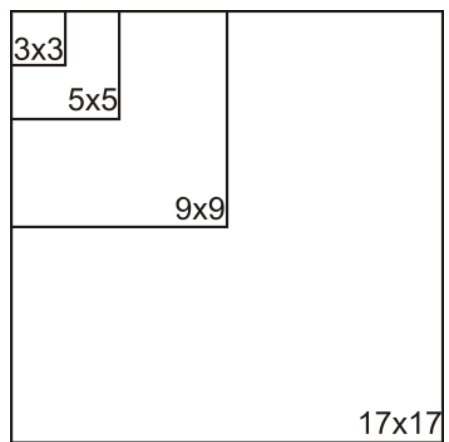

Fig. 10. Measuring fields for data reduction.

\section{VALIDATION OF RESULTS}

From the remaining measurement data after data reduction, we obtain triangles by means of triangulation which differ from the previously expected triangles (see Fig. 9). Therefore, in the end it is necessary to examine the complete surface of the CAD model. For this purpose, an equidistant measurement data set, which in its size corresponds to the original matrix, is created from the triangulated surface. Then, the difference to the original measured value is calculated for every triangulated measured value. Afterwards these differences are added in the column. This way, it is possible to describe the accuracy of the created CAD model and, therefore, the whole method described above. Fig. 11 shows a section of the added error with 2600 measured values per column. To be able to refer the error values to the specific areas of the riblet structures two periods of the riblet structures are schematically depicted.

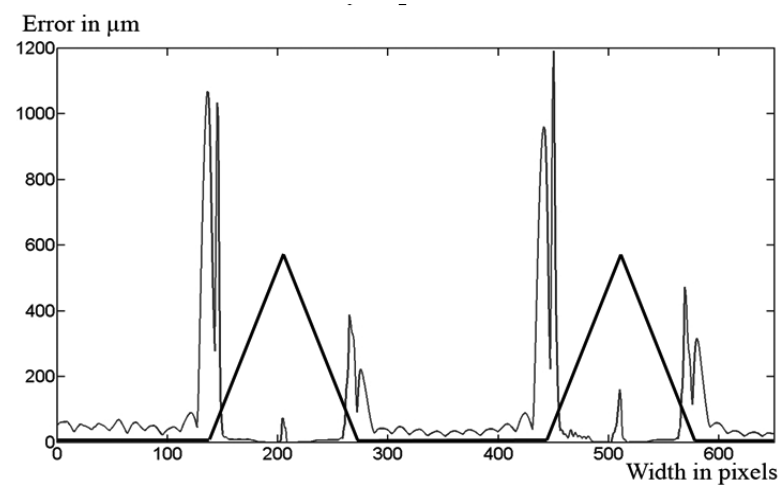

Fig. 11. Error visualization in columns.

The results show that the required accuracy, particularly in the tip area, is achieved. Even in the unimportant bottom area of riblets there is only an error of approx. $0.5 \mu \mathrm{m}$ per pixel. This error is approx. $0.08 \mu \mathrm{m}$ in the more important tip area what is considered to be low enough since the tip radius lays around $1 \mu \mathrm{m}$. Since the whole structure is depicted with triangles a quality rating of all triangles is introduced. Therefore the smallest internal angle of each triangle is determined and the result is shown in the following histogram in Fig. 12.

The used data set contains 3.2 million triangles while the main part of all triangles has a smallest angle of $45^{\circ}$ what is considered to be a high quality element.

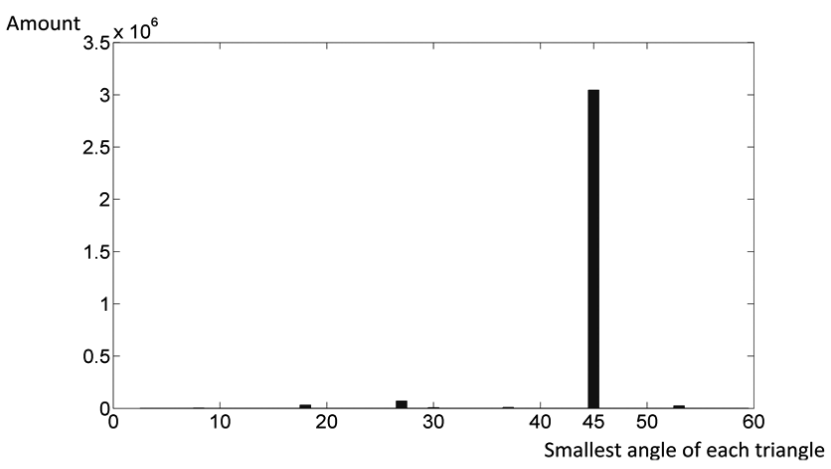

Fig. 12. Histogram for triangle quality.

\section{CONCLUSION}

In order to analyze the quality of the riblet-structured surfaces, it is necessary to carry out measurements in the oil passages on the upscaled models of the structures. For this purpose, measurement data sets from positive molds of the original structure are created by means of a confocal microscope. The processing of these measurement data is described in this paper. The processing includes the elimination of the stitching errors caused by the microscope software and the new alignment of the created measurement data sets. Other chapters present methods for the elimination of measurement artifacts and mold errors. After the application of these methods, the newly adjusted and error-free measurement data sets are added to a large measuring field. Due to the large attention to detail of the 
application of these methods, the newly adjusted and error-free measurement data sets are added to a large measuring field. Due to the large attention to detail of the measurement data and the extensive area of the measuring field, the obtained CAD files will require an enormous disk space. For this reason, irrelevant measuring points are eliminated from the surface representation. The remaining measuring points are connected by means of the triangulation method and are exported as CAD files. Finally, there is an examination of the accuracy and the triangulation quality of the results, which shows that all the applied measures have a negligibly small influence on the quality of the measurement data.

\section{OUTLOOK}

In the course of the described method, individual measurements are repeatedly added; sometimes measurement data are lost in the overlapping area. Therefore, it would make sense to develop a new stitching algorithm which will be implemented very early in this process, in the best case already when carrying out individual measurements. This will make it possible to have a significantly larger number of the original measured values in the CAD model, while having the same number of the individual measurements.

Another improvement can be carried out in regard to the detection of surface defects. By means of a comparison of the height values on a color image of the surface, it is possible to better decide which surface defects are created by the measurement method and which are part of the riblet structure.

\section{REFERENCES}

[1] W. Hage, "Zur widerstandsverminderung von dreidimensionalen riblet-strukturen und anderen oberflächen," Technische Universität Berlin, Dissertation, 2004.

[2] C. Lietmeyer, "Berechnungsmodell zur Widerstandsbeeinflussung nicht-idealer Riblets auf Verdichterschaufeln," Leibniz Universität Hannover, Dissertation, 2013.

[3] C. Lietmeyer, B. Denkena, R. Kling, T. Krawczyk, L. Overmeyer, E. Reithmeier, R. Scheuer, T. Vynnyk, B. Wojakowski, and J. R. Seume, "Recent Advances in Manufacturing of Riblets on Compressor Blades and their Aerodynamic Impact," Journal of Turbomachinery, Article Number: TURBO-12-1136, 2013.

[4] T. Vynnyk, "REM topografiemessungen an mikro- und nanostrukturierten oberflächen," Shaker Verlag, Aachen, Dissertation 2010.

[5] B. Delaunay, "Sur la sphère vide. A la mémoire de georges voronoi," Bulletin de l'Académie des Sciences de l 'URSS, no. 6, pp. 793-800, 1934.

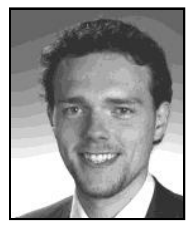

R. Scheuer received his Diplom-Ingenieur degree in 2010 and works since that time at the Institute of Measurement and Automatic Control as a research associate in the field of Industrial and Medical Imaging.

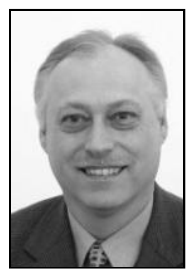

E. Reithmeier is the director of the Institute of Measurement and Automatic Control at the Leibniz Universitaet Hannover since 1996.

The main research fields are Production Metrology, Industrial and Medical Imaging, Control Engineering and Acoustics

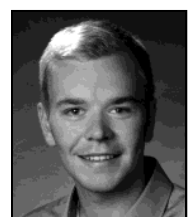

N. B. Windeler is a postgraduate student at the Leibniz Universitaet Hannover. Since 2012 he works as an assistant at the Institute of Measurement and Automatic Control. 